Pacific Journal of Mathematics

BANACH ALGEBRAS ASSOCIATED WITH SPHERICAL
REPRESENTATIONS OF THE FREE GROUP 


\title{
BANACH ALGEBRAS ASSOCIATED WITH SPHERICAL REPRESENTATIONS OF THE FREE GROUP
}

\author{
RYSZARD SZWARC
}

\begin{abstract}
We prove that any spherical representation of the free group $\mathbb{F}$ weakly contains the regular representation. Moreover $C_{\pi}^{*}$, the $C^{*}$ algebra associated with the spherical representation $\pi$, is a compact extension of the reduced $C^{*}$-algebra of $\mathbb{F}$. We also show that the standard projection onto radial functions admits extensions to $C_{\pi}^{*}$ for a class of representations $\pi$ of $\mathbb{F}$ which includes spherical representations, as well as the regular representation and the universal representation.
\end{abstract}

Introduction. Let $\mathbb{F}_{r}$ be a free group on $r$ generators $x_{1}, \ldots, x_{r}$. Let $\mu_{1}$ be the finitely supported probability measure equidistributed on $\left\{x_{1}^{ \pm 1}, x_{2}^{ \pm 1}, \ldots, x_{r}^{ \pm 1}\right\}$. The operator of convolution by $\mu_{1}$ is the analogue of the Laplace-Beltrami operator on Riemann rank one symmetric spaces. By [11] the $l^{1}$-spectrum of $\mu_{1}$ can be identified with the ellipse $E=\left\{z=x+i y: x^{2}+\left(\frac{r}{r-1} y\right)^{2} \leq 1\right)$. Any point $z$ of $E$ corresponds in one-to-one fashion to a spherical function $\phi_{z}$ the eigenfunction of $\mu_{1}$ with eigenvalue $z$. We refer to [11], [7] for this subject.

For real $z$ spherical functions are positive definite and give rise to unitary representations of $\mathbb{F}_{r}$. Basing our argument on a particular realisation of these representations and on the simplicity of the reduced $C^{*}$-algebra of $\mathbb{F}_{r}[\mathbf{1 0}]$, we prove that all spherical representations weakly contain the regular representation. Moreover the $C^{*}$ algebras associated with spherical representations are compact extensions of $C_{\text {red }}^{*}\left(\mathbb{F}_{r}\right)$, the $C^{*}$-algebra associated with the regular representation.

Finally we consider the standard projection onto radial functions on $\mathbb{F}_{r}$ and we prove that it is bounded on any $C^{*}$-algebra associated with spherical functions.

Acknowledgment. I am grateful to Uffe Haagerup for suggesting the problem and for helpful discussions.

Spherical representations. Let $\mathbb{F}_{r}$ be a free group on $r$ generators $x_{1}, x_{2}, \ldots, x_{r}, r \geq 2$. Any element $x$ of $\mathbb{F}_{r}$ may be uniquely ex- 
pressed as a reduced word in $x_{1}, \ldots, x_{r}$ and their inverses. The number of letters of this reduced word is called the length of $x$ and is denoted by $|x|$.

A complex function on $\mathbb{F}_{r}$ which values depend only on the length $|x|$ will be called radial. They are the analogues of bi- $K$-invariant functions on $\operatorname{SL}(2, \mathbb{R})$. The space $l_{\#}^{1}$ of absolutely summable radial functions forms a commutative Banach algebra with respect to convolution operation. This is due to the fact that if $\chi_{n}$ is defined as $\chi_{n}(x)=1$ if $|x|=n$ and 0 otherwise, then:

$$
\begin{aligned}
& \chi_{1}^{*} \chi_{n}=\chi_{n+1}+(2 r-1) \chi_{n-1}, \quad n \geq 2 \\
& \chi_{1}^{*} \chi_{1}=\chi_{2}+2 r \chi_{0}
\end{aligned}
$$

(see [3], [4], [11]). In particular it means that $l_{\#}^{1}$ is generated by the function $\chi_{1}$. The Gelfand space of $l_{\#}^{1}$, when identified with the $l^{1}$ spectrum of $\chi_{1}$, coincides ([11], Theorem 2.1) with the closed ellipse:

$$
E=\left\{z=x+i y \in \mathbb{C}:\left(\frac{x}{2 r}\right)^{2}+\left(\frac{y}{2 r-2}\right)^{2} \leq 1\right\} .
$$

Moreover multiplicative functionals on $l_{\#}^{1}$ are given by

$$
l_{\#}^{1} \ni f \longmapsto\left\langle f, \bar{\phi}_{z}\right\rangle=\sum_{x \in \mathbb{F}_{r}} f(x) \phi_{z}(x),
$$

where $\phi_{z}, \quad z \in E$, are bounded complex functions with properties:

(i) $\phi_{z}(e)=1$;

(ii) $\chi_{z}^{*} \phi_{z}=z \phi_{z}$;

(iii) $\phi_{z}$ is radial.

In analogy to $\operatorname{SL}(2, \mathbb{R})$ such functions are called spherical (see [3], [7]) and the properties (i), (ii), (iii) determine $\phi_{z}$ uniquely. Explicit formulas expressing $\phi_{z}$ can be found in [7], [11] and [13].

The theory developed in the papers [11], [7], [9] (see also [13]) gives that for any point $z$ of the interior of $E$ (including also two points $2 r$ and $(-2 r)$ which correspond to the only characters $x \mapsto 1$ and $x \mapsto(-1)^{|x|}$ on $\mathbb{F}_{r}$ ) the spherical function $\phi_{z}$ occurs as the matrix coefficient of a uniformly bounded representation of $\mathbb{F}_{r}$. The real segment $[-2 r, 2 r]$ corresponds to all unitary representations: in particular the segment $[-2 \sqrt{2 r-1}, 2 \sqrt{2 r-1}]$ (i.e. the $l^{2}$-spectrum of convolution operator by $\chi_{1}$ ) corresponds to the principal series while the two remaining parts $[-2 r,-2 \sqrt{2 r-1})$ and $(2 \sqrt{2 r-1}, 2 r]$ form the complementary series. 
In the sequel we will need a realisation of spherical representations given in [13] because of its good additional features. In [13] the spherical functions are parametrized by the annulus $A=\{z \in$ $\left.\mathbb{C}:(2 r-1)^{-1} \leq|z| \leq 1\right\}$ which can be mapped onto $E$ by the function $\gamma(z)=(2 r-1) z+\frac{1}{z}$. Taking it into account we reformulate below the main results of [13] with respect to the ellipse-parametrization.

THEOREM 1 ([12], Theorem 8 and remarks following it). Let $\mathbb{F}_{r}$ be a free group on $r$ generators. There exists an analytic series of uniformly bounded representations $\pi_{z}, z \in E=\left\{x+i y:\left(\frac{x}{2 r}\right)^{2}+\left(\frac{y}{2 r-2}\right)^{2} \leq 1\right\}$, on a Hilbert space $\mathscr{H}$ and a unit vector $\zeta \in \mathscr{H}$ such that:

(i) $\left\langle\pi_{z}(x) \zeta, \zeta\right\rangle=\phi_{z}(x), x \in \mathbb{F}_{r}$;

(ii) $\pi_{z}(x)^{*}=\pi_{\bar{z}}(x)^{-1}, x \in \mathbb{F}_{r}$;

(iii) $\pi_{z}(x)-\pi_{z^{\prime}}(x)$ has finite rank for any $z, z^{\prime} \in E$ and $x \in \mathbb{F}_{r}$;

(iv) Any representation $\pi_{z}$ leaves no nontrivial subspace invariant and representations $\pi_{z}$ are mutually inequivalent;

(v) $\pi_{z}$ is unitary if and only if $z \in \mathbb{R}$. Otherwise $\pi_{z}$ cannot be made unitary by introducing equivalent inner product.

The series $[-2 \sqrt{2 r-1}, 2 \sqrt{2 r-1}]$ occurs in decomposition of the regular representation (see [11], Theorem 6.2). That is why it is called the principal series. Remaining parts of $[-2 r, 2 r]$ are called the complementary series.

The aim of the paper is to show that any representation of complementary series, as well as of principal series, weakly contains the regular representation. In some sense (see Lemma 2) we also prove it for nonunitary spherical representations. The crucial are Theorem 1 (iii) and the Powers' theorem which states that $C_{\text {red }}^{*}\left(\mathbb{F}_{r}\right)$, the reduced $C^{*}$-algebra of $\mathbb{F}_{r}$, is simple i.e. contains no nontrivial two-sided ideals.

Let $\lambda$ denote the left regular representation of $\mathbb{F}_{r}$. By Powers' theorem any unitary representation weakly contained in $\lambda$ is weakly equivalent to $\lambda$.

LEMMA 1. Let $(\pi, \mathscr{H})$ be a unitary representation of $\mathbb{F}_{r}$ weakly contained in the regular representation. Then for any compact operator $K$ on $\mathscr{H}$ and any $f \in l^{1}\left(\mathbb{F}_{r}\right)$ we have:

$$
\|\pi(f)+K\| \geq\|\pi(f)\| .
$$

Proof. Let $C_{\pi}^{*}$ be the $C^{*}$-algebra associated with $\pi$. Then by remarks preceding the lemma $C_{\pi}^{*}$ is isometrically isomorphic to $C_{\mathrm{red}}^{*}\left(\mathbb{F}_{r}\right)$. 
Hence by [10] it is a simple $C^{*}$-algebra. Consider the homomorphism of $C_{\pi}^{*}$ into the Calkin algebra $\mathscr{B}(\mathscr{H}) / \mathscr{K}(\mathscr{H})$ (where $\mathscr{K}(\mathscr{H})$ denotes the set of all linear compact operators on $\mathscr{H})$ given on $l^{1}\left(\mathbb{F}_{r}\right)$ as

$$
l^{1}\left(\mathbb{F}_{r}\right) \ni f \mapsto \pi(f)+\mathscr{K}(\mathscr{H}) \in \mathscr{B}(\mathscr{H}) / \mathscr{K}(\mathscr{H}) .
$$

Because $C_{\pi}^{*}$ has no ideals then this mapping is an isometry. Thus $\inf _{K \in \mathscr{K}(\mathscr{H})}\|\pi(f)+K\|=\|\pi(f)\|$ which proves the lemma.

Lemma 2. Let $z \in E$ and $f \in l^{1}\left(\mathbb{F}_{r}\right)$. Then $\left\|\pi_{z}(f)\right\| \geq\|\lambda(f)\|$. In particular any spherical representation $\pi_{z}, z \in E$ has trivial kernel in $l^{1}\left(\mathbb{F}_{r}\right)$.

Proof. Let us apply Lemma 1 to a representation of the principal series e.g. $\pi_{0}$. By Theorem 1 (iii) the operator $k=\pi_{z}(f)-\pi_{0}(f)$ is compact whatever $f$ from $l^{1}\left(\mathbb{F}_{r}\right)$ is. Therefore

$$
\left\|\pi_{z}(f)\right\|=\left\|\pi_{0}(f)+K\right\| \geq\left\|\pi_{0}(f)\right\|=\|\lambda(f)\|
$$

because $\pi_{0}$ and $\lambda$ are weakly equivalent.

Lemma 2 implies immediately:

THEOREM 2. Any representation $\pi_{z}$ of the complementary series of $\mathbb{F}_{r}$, except two characters $z= \pm 2 r$, contains weakly the regular representation.

For any $z \in E$ let $C_{\pi}$ denote the completion of $l^{1}\left(\mathbb{F}_{r}\right)$ with respect to the norm $\|f\|_{C_{\pi_{z}}}=\left\|\pi_{z}(f)\right\|$. Then $C_{\pi_{z}}$ is a Banach algebra which becomes a $C^{*}$-algebra whenever $\pi_{z}$ is unitary i.e. $z \in[-2 r, 2 r]$.

THEOREM 3. Let $z \in \int E$. Then the identity map $f \mapsto f$ on $l^{1}\left(\mathbb{F}_{r}\right)$ extends to an epimorphism from $C_{\pi}$ onto $C_{\text {red }}^{*}\left(\mathbb{F}_{r}\right)$. Moreover the kernel of this map is isomorphic to the ideal of all compact operators on a Hilbert space.

Proof. The first part follows from Lemma 2. Next observe that $C_{\pi_{z}}$ is isometrically isomorphic to the norm closure of $\left\{\pi_{z}(f): f \in\right.$ $\left.l^{1}\left(\mathbb{F}_{r}\right)\right\}$ in $\mathscr{B}(\mathscr{H})$ because $\pi_{0}$ and $\lambda$ are weakly equivalent. Thus we have to determine the kernel of the homomorphism between these two subalgebras of $\mathscr{B}(\mathscr{H})$ given by:

$$
\pi_{z}(f) \longmapsto \pi_{0}(f), \quad f \in l^{1}\left(\mathbb{F}_{r}\right) .
$$


Let $\pi_{z}\left(f_{n}\right)$ be a Cauchy sequence in $B(\mathscr{H})$ such that $\pi_{0}\left(f_{n}\right) \rightarrow 0$. Hence $\pi_{z}\left(f_{n}\right)=\pi_{0}\left(f_{n}\right)+K_{n}$, where $K_{n}, n=1,2, \ldots$, are compact operators (see Theorem 1 (iii)). This yields $\lim _{n} \pi_{z}\left(f_{n}\right)=\lim _{n} K_{n}$ is a compact operator. It means that the kernel is contained in $\mathscr{K}(\mathscr{H})$. On the other hand $C_{\pi}$. contains $\mathscr{K}(\mathscr{H})$ by [13] (Remark following Proposition 2). Moreover the image of $\mathscr{K}(\mathscr{H})$ under this homomorphism must be trivial because it is a two-sided ideal in $C_{\pi_{0}}^{*}$.

REMARK. Theorem 2 implies that $C_{\pi}$ are compact extensions of the reduced $C^{*}$-algebra of $\mathbb{F}_{r}$ in the sense of [2] (see also [6]). All these extensions are trivial (see [2] for the definition) because they coincide with the trivial extension corresponding to a representation of the principal series.

Proposition 1. Let $z \in E$. Then the spectrum of the operator $\pi_{z}\left(\chi_{1}\right)$ coincides with $\{z\} \cup[-2 \sqrt{2 r-1}, 2 \sqrt{2 r-1}]$.

Proof. By [13] (Theorem 5) the spectrum of $\pi_{z}\left(\chi_{1}\right)$ consists of $\{z\}$ and a subset of $[-2 \sqrt{2 r-1}, 2 \sqrt{2 r-1}]$ (recall that we use another parametrization). Thus we should prove only that the entire interval enters into the spectrum. However it follows from the fact that $\pi_{z}\left(\chi_{1}\right)$ is a finite dimensional perturbation of the selfadjoint operator $\pi_{0}\left(\chi_{1}\right)$ which spectrum coincides with the spectrum of $\lambda\left(\chi_{1}\right)$ in $C_{\text {red }}^{*}\left(\mathbb{F}_{r}\right)$ (because $\pi_{0}$ and $\lambda$ are weakly equivalent) i.e. with the interval $[-2 \sqrt{2 r-1}, 2 \sqrt{2 r-1}]$.

Conditional expectation related to radial functions. As we have seen before the radial functions on $\mathbb{F}_{r}$ form a commutative convolution algebra. There is a natural projection $\mathscr{E}$ from the set of all complex function on $\mathbb{F}_{r}$ onto radial functions:

$$
\mathscr{E} f(x)=\frac{1}{\#\left\{x \in \mathbb{F}_{r}:|x|=n\right\}} \sum_{|x|=n} f(x) .
$$

The operator $\mathscr{E}$ satisfies (cf. [11], [5])

(i) $\mathscr{E} f=f$ if and only if $f$ is radial;

(ii) $\mathscr{E}(f * \mathscr{E} g)=\mathscr{E}(\mathscr{E} f * g)=\mathscr{E}(f * \mathscr{E} g)$;

(iii) $\mathscr{E} f(e)=f(e)$.

These properties determine $\mathscr{E}$ uniquely. By general theorems concerning weak conditional expectations $\mathscr{E}$ extends to the contraction on the von Neumann algebra $\mathrm{VN}\left(\mathbb{F}_{r}\right)$ of $\mathbb{F}_{r}$ (see [5]). 
Let $\pi$ be a unitary representation of $\mathbb{F}_{r}$ without kernel in $l^{1}\left(\mathbb{F}_{r}\right)$. There arises a question whether the operator $\mathscr{E}$ can extend from $l^{1}\left(\mathbb{F}_{r}\right)$ to a contraction on $C_{\pi}^{*}\left(\mathbb{F}_{r}\right)$.

Proposition 2. Let $\pi$ be a unitary representation of $\mathbb{F}_{r}$. Assume that $\pi$ contains weakly all spherical representations $\pi_{t}$ such that $t$ belongs to the spectrum of the operator $\pi\left(\chi_{1}\right)$. Then $\mathscr{E}$ extends by continuity to a contraction on $C_{\pi}^{*}\left(\mathbb{F}_{r}\right)$.

Proof. It suffices to show that for any function $f$ in $l^{1}\left(\mathbb{F}_{r}\right)$ there holds $\|\pi(\mathscr{E} f)\| \leq\|\pi(f)\|$. Denote by $\mathscr{A}$ the unital $C^{*}$-algebra generated by $\pi\left(\chi_{z}\right)$. If $t \in \sigma\left(\pi\left(\chi_{1}\right)\right)$ then by (2), the Gelfand-Naimark theorem and Theorem 2 (i) we have

$$
\begin{aligned}
\|\pi(\mathscr{E} f)\| & =\sup _{t \in \sigma\left(\pi\left(\chi_{1}\right)\right)}\left|\left\langle\mathscr{E} f, \phi_{t}\right\rangle\right|=\sup _{t \in \sigma\left(\pi\left(\chi_{1}\right)\right)}\left|\left\langle f, \phi_{t}\right\rangle\right| \\
& =\sup _{t \in \sigma\left(\pi\left(\chi_{1}\right)\right)}\left|\left\langle\pi_{t}(f) \zeta, \zeta\right\rangle\right| \\
& \leq \sup _{t \in \sigma\left(\pi\left(\chi_{1}\right)\right)}\left\|\pi_{t}(f)\right\| \leq\|\pi(f)\|
\end{aligned}
$$

(the second inequality holds because $\phi_{t}$ are radial while the last inequality follows from the assumptions).

The next theorem is already known for the regular (see [11], [5]) and the universal representation (see [8], Lemma B).

THEOREM 4. Let $\pi$ be one of the representations of the free group $\mathbb{F}_{r}$ : regular, universal or spherical unitary representation. Then the projection $\mathscr{E}$ onto radial functions can be extended from $l^{1}\left(\mathbb{F}_{r}\right)$ to the $C^{*}$-algebra $C_{\pi}^{*}$ associated with $\pi$.

Proof. It suffices to check that any of the mentioned representations satisfies the hypothesis of Proposition 2. Clearly it is valid for the regular and universal representations. By Powers' theorem it also holds for the principal series. Finally the case of complementary series follows from Proposition 1 and Theorem 2.

EXAMPLE. Theorem 3 does not hold for any unitary representation of $\mathbb{F}_{r}$ which lifts to a faithful representation of $l^{1}\left(\mathbb{F}_{r}\right)$. In fact, let $\mathbb{F}_{r}=$ $g p\left\{x_{1}, x_{2}, \ldots, x_{r}\right\}$ and let $\pi$ be a one-dimensional representation given by $\pi\left(x_{1}\right)=-1, \pi\left(x_{2}\right)=\cdots=\pi\left(x_{r}\right)=1$. Put $\Pi=\pi \oplus \lambda$ and let $f=\delta_{x_{1}}+\delta_{x_{2}}+\delta_{x_{1}^{-1}}+\delta_{x_{2}^{-1}}$. Thus $\Pi(f)=0 \oplus \lambda(f)$ while

$$
\Pi(\mathscr{E} f)=\frac{4(r-2)}{r} \oplus \frac{2}{r} \lambda\left(\chi_{1}\right) \text {. }
$$


Therefore by [1] (Theorem IV J) we have

and

$$
\|\Pi(\mathscr{E} f)\|=\max \left\{\frac{4(r-2)}{r}, \frac{4 \sqrt{2 r-1}}{r}\right\} \rightarrow 4, \quad \text { when } r \rightarrow \infty
$$

$$
\|\pi(f)\|=2 \sqrt{3}<4 .
$$

\section{REFERENCES}

[1] C. A. Akemann and P. A. Ostrand, Computing norms in group $C^{*}$-algebras, Amer. J. Math., 98 (1976), 1015-1047.

[2] W. Arveson, Notes on extensions of $C^{*}$-algebras, Duke Math. J., 44 (1976), 329-355.

[3] P. Cartier, Fonctions harmoniques sur un arbre, Symposia Math., 9 (1972), 203-270.

[4] J. M. Cohen, Operator norms on free groups, Boll. Un. Math. Ital., 1-B (1982), 1055-1065.

[5] J. M. Cohen, and L. De Michele, The radial Fourier-Stieltjes algebra of free groups, Contemporary Math., 10 (1982), 33-40.

[6] R. G. Douglas, $C^{*}$-algebra extensions and K-homology, Annals of Math. Studies 95, Princeton Univ. Press 1980.

[7] A. Figà-Talamanca, and M. A. Picardello, Spherical functions and harmonic analysis on free groups, J. Funct. Anal., 47 (1982), 281-304.

[8] U. Haagerup, $M_{0} A(G)$-functions which are not coefficients of uniformly bounded representations, manuscript 1985.

[9] A. M. Mantero and A. Zappa, The Poisson transform on free groups and uniformly bounded representations, J. Funct. Anal., 51 (1983), 372-400.

[10] R. T. Powers, Simplicity of the $C^{*}$-algebra associated with the free group on two generators, Duke. Math. J., 42 (1975), 151-156.

[11] T. Pytlik, Radial functions on free group and a decomposition of the regular representation into irreducible components, J. Reine Angew. Math., 326 (1981). 124-135.

[12] T. Pytlik, and R. Szwarc, An analytic family of uniformly bounded representations of free groups, Acta Math., 157 (1986), 287-309.

[13] R. Szwarc, An analytic series of irreducible representations of the free group, Ann. Inst. Fourier (Grenoble), 38 (1988), 87-110.

Received June 8, 1988 and, in revised form November 5, 1988. This paper was written while the author held a C.N.R. fellowship at the University of Rome "La Sapienza".

WROCLAW UNIVERSITY

PL. GRUNWALDZKI $2 / 4$

PL 50-384 Wroclaw, Poland

AND

Istituto "CASTELnUOVo"

UNIVERsità "La SAPIENZA"

Piazzale A. Moro 2

I-00185 Roma, Italy 



\section{PACIFIC JOURNAL OF MATHEMATICS EDITORS}

\author{
V. S. VARADARAJAN \\ (Managing Editor) \\ University of California \\ Los Angeles, CA 90024-1555-05 \\ Herbert Clemens \\ University of Utah \\ Salt Lake City, UT 84112 \\ Thomas ENRIGHT \\ University of California, San Diego \\ La Jolla, CA 92093
}

R. FINN

Stanford University

Stanford, CA 94305

Hermann FlaschKa

University of Arizona

Tucson, AZ 85721

VAughan F. R. Jones

University of California

Berkeley, CA 94720

Steven Kerckhoff

Stanford University

Stanford, CA 94305
RobION KIRBY

University of California

Berkeley, CA 94720

C. C. MOore

University of California

Berkeley, CA 94720

HAROLd STARK

University of California, San Diego La Jolla, CA 92093

\begin{tabular}{|c|c|c|c|c|}
\hline & ASSOCIA & E EDI & & \\
\hline R. ARENS & $\begin{array}{l}\text { E. F. BECKENBACH } \\
(1906-1982)\end{array}$ & NeumanN & $\begin{array}{c}\text { F. WoLF } \\
(1904-1989)\end{array}$ & K. Yoshida \\
\hline & SUPPORTINC & INSTI & TIONS & \\
\hline UNIVERSITY & ARIZONA & UNIVERS & OF OREGON & \\
\hline UNIVERSITY & BRITISH COLUMBIA & UNIVER & OF SOUTHEI & CALIFORNIA \\
\hline CALIFORNIA & STITUTE OF TECHNOLOGY & STANFOI & UNIVERSITY & \\
\hline UNIVERSITY & CALIFORNIA & UNIVER & OF HAWAII & \\
\hline MONTANA S & TE UNIVERSITY & UNIVER & OF TOKYO & \\
\hline UNIVERSITY & NEVADA, RENO & UNIVER & OF UTAH & \\
\hline NEW MEXIC & TATE UNIVERSITY & WA & N STATE UN & ERSITY \\
\hline OREGON ST & UNIVERSITY & & OF WASHINC & $\mathrm{ON}$ \\
\hline
\end{tabular}




\section{Pacific Journal of Mathematics}

\section{Vol. 143, No. $1 \quad$ March, 1990}

Walter Bergweiler, On the fix-points of composite functions $\ldots \ldots \ldots \ldots \ldots 1$ Aldo Biancofiore, Maria Lucia Fania and Antonio Lanteri, Polarized surfaces with hyperelliptic sections $\ldots \ldots \ldots \ldots \ldots \ldots \ldots \ldots \ldots$

Ciprian Borcea, Deforming varieties of $k$-planes of projective complete

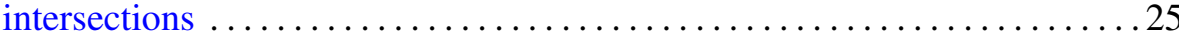

Morton Brown, Fixed points for orientation preserving homeomorphisms of

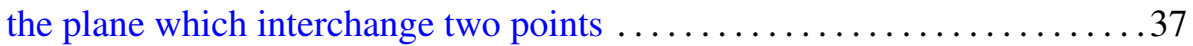

Hao Zhi Chuan, Note on the inequality of the arithmetic and geometric means

Paul Henry Edelman and Dennis E. White, Codes, transforms and the

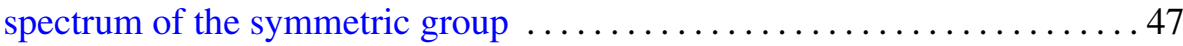

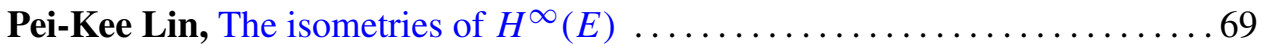

James J. Moloney, Residue class domains of the ring of convergent

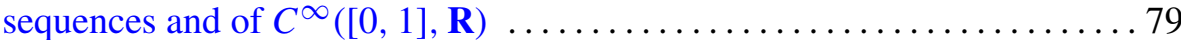

Zhong-Jin Ruan, The structure of pure completely bounded and completely

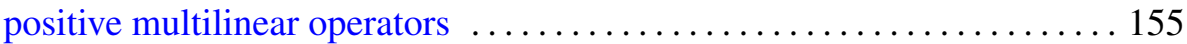

Wolfgang Ruess and William H. Summers, Weakly almost periodic semigroups of operators

Gideon Schwarz, A pretender to the title "canonical Moebius strip" . . . . . . 195

Ryszard Szwarc, Banach algebras associated with spherical representations of the free group 